\title{
Seed-mediated Growth of Gold Nanocrystals: Changes to the Crystallinity or Morphology as Induced by the Treatment of Seeds with a Sulfur Species
}

Yiqun Zheng, ${ }^{\dagger, ~} \S$ Ming Luo, ${ }^{\ddagger} \|, \S$ Jing Tao, ${ }^{\perp}$ Hsin-Chieh Peng, ${ }^{\dagger}$ Dehui Wan, ${ }^{\ddagger}$ Yimei Zhu, ${ }^{\perp}$ and Younan $\mathrm{Xia}^{*}, \dagger, \ddagger$

${ }^{\dagger}$ School of Chemistry and Biochemistry, Georgia Institute of Technology, Atlanta, Georgia 30332, United States

${ }^{\ddagger}$ The Wallace H. Coulter Department of Biomedical Engineering, Georgia Institute of Technology and Emory University, Atlanta, Georgia 30332, United States

"Key Laboratory of Analytical Chemistry for Biology and Medicine, Ministry of Education, College of Chemistry and Molecular Sciences, Wuhan University, Wuhan 430072, P. R. China

${ }^{\perp}$ Condensed Matter Physics and Materials Science Department, Brookhaven National Laboratory, Upton, New York 11973, United States

$\S$ These two authors contributed equally to this work. 


\begin{abstract}
We report our observation of changes to the crystallinity or morphology during seedmediated growth of Au nanocrystals. When single-crystal Au seeds with a spherical or rodlike shape were treated with a chemical species such as $\mathrm{S}_{2} \mathrm{O}_{3}{ }^{2-}$ ions, twin defects were developed during the growth process to generate multiply twinned nanostructures. X-ray photoelectron spectroscopy analysis indicated that the $\mathrm{S}_{2} \mathrm{O}_{3}{ }^{2-}$ ions were chemisorbed on the surfaces of the seeds during the treatment. The chemisorbed $\mathrm{S}_{2} \mathrm{O}_{3}{ }^{2-}$ ions somehow influenced the crystallization of Au atoms added onto the surface during a growth process, leading to the formation of twin defects. In contrast to the spherical and rod-like Au seeds, the single-crystal structure was retained to generate a concave morphology when single-crystal Au seeds with a cubic or octahedral shape were used for a similar treatment and then seed-mediated growth. The different outcomes are likely related to the difference in spatial distribution of $\mathrm{S}_{2} \mathrm{O}_{3}{ }^{2-}$ ions chemisorbed on the surface of a seed. This approach based on surface modification is potentially extendable to other noble metals for engineering the crystallinity and morphology of nanocrystals formed via seed-mediated growth.
\end{abstract}

KEYWORDS: Crystallinity · gold · thiosulfate ions $\cdot$ seed-mediated growth $\cdot$ twin defects 


\section{INTRODUCTION}

Crystallinity and morphology are two important attributes of nanocrystals as they may affect the catalytic, optical, and electrical properties of these nanocrystals. ${ }^{1}$ Generally, crystallinity is referred to as the degree of structural order in a solid. For a single crystal, all the atoms are arranged in a perfect lattice with translational, long-range periodicity along all directions. The periodic structure can be disrupted locally when defects are introduced. ${ }^{2}$ In this case, the arrangement of atoms in certain regions can lose the periodicity along at least one direction, which reduces the degree of structural perfection and thus leads to a change in crystallinity. Among various types of defects, twin is a common planar defect for face-centered cubic ( $f c c)$ metals. It is like a mirror plane, where arrangement of atoms on one side is a perfect reflection of what is on the other side. ${ }^{3}$ Depending on the number and locations of twin defects in the product, the crystallinity of noble-metal nanocrystals can be classified into three major categories: single crystal, singly twinned, and multiply twinned. ${ }^{4}$ Products with different degrees of crystallinity may exhibit a myriad of shapes and surface structures in addition to the difference in internal structure. ${ }^{5,6}$ All these variations, as induced by the difference in crystallinity, can exert a major impact on their physicochemical properties and potential applications in fields such as optics, mechanics, electronics, and catalysis. ${ }^{7-14}$ To this end, it is of critical importance to have an ability to manipulate the crystallinity of noblemetal nanocrystals. ${ }^{15-18}$

Although noble-metal nanocrystals with different degrees of crystallinity may co-exist in the final products of a typical synthesis, it should be pointed out that the evolution of twin defects from single-crystal seeds during the growth may not be favored by thermodynamics. This is because the presence of defects will break the intrinsic cubic symmetry of the lattice and thus increase the total free energy of the system. ${ }^{19}$ In addition, the defect sites often bring in extra strain energy and thus can be more susceptible to corrosive environments involving species for oxidative etching. ${ }^{20}$ Therefore, when single-crystal seeds are used for growth to obtain larger nanocrystals, the resultant products may exhibit differences in terms of size and shape but they generally will preserve the single crystallinity of the seeds. ${ }^{21-25}$ This principle has been widely accepted and routinely used to direct the growth of noble-metal nanocrystals with a large number of different shapes and thus facets on the surface. ${ }^{26}$

Despite the aforementioned believing, recent work indicated that change in crystallinity between the seed and the final product could take place during the seed-mediated growth of 
binary noble-metal nanocrystals. This can be attributed to the difference in intrinsic properties between the two distinctive metals, including the lattice constant and bond energy, among others. For example, Pt-Au penta-twinned nanorods and Au-Ag multi-twinned icosahedrons have been obtained from seeds made of Pt or Au polyhedral particles with a single-crystal structure. $^{27,28}$ However, when the same metal is used for seed-mediated growth, there has been no report on the possible change in crystallinity from single-crystal seeds to multiply twinned products.

Here we wish to report our recent observations on changes to crystallinity during the seed-mediated growth of Au on single-crystal Au seeds. In particular, when single-crystal Au seeds with a spherical or rod-like shape were treated with a chemical species containing sulfur, twin defects would develop during the growth process to yield multiply twinned nanocrystals. Interestingly, it was found that this phenomenon depended on the shape of the single-crystal Au seeds. In contrast to the spherical and rod-like seeds, concave structures rather than twin detects were observed to develop during the growth process when Au singlecrystal seeds with a cubic or octahedral shape were used.

\section{MATERIALS AND METHODS}

Materials. Ascorbic acid (AA, $\geq 99.0 \%$ ), gold(III) chloride trihydrate $\left(\mathrm{HAuCl}_{4} \cdot 3 \mathrm{H}_{2} \mathrm{O}\right.$, $\geq 99.9 \%$ ), sodium borohydride $\left(\mathrm{NaBH}_{4}, 98 \%\right)$, sodium thiosulfate $\left(\mathrm{Na}_{2} \mathrm{~S}_{2} \mathrm{O}_{3}, 99 \%\right)$, sodium sulfate $\left(\mathrm{Na}_{2} \mathrm{SO}_{4}, \geq 99.0 \%\right)$, and hexadecyltrimethylammonium bromide (CTAB, $\left.\geq 99 \%\right)$, hexadecyltrimethylammonium chloride (CTAC, $\geq 98.0 \%$ ) were all obtained from SigmaAldrich and used as received. Sodium sulfide nonahydrate $\left(\mathrm{Na}_{2} \mathrm{~S} \cdot 9 \mathrm{H}_{2} \mathrm{O}, 98.0 \%\right)$ was obtained from Spectrum Chemical Manufacturing Corporation. Sulfur powder (precipitated, 99.5\%) was obtained from Alfa Aesar. Deionized water with a resistivity of $18.2 \mathrm{M} \Omega \bullet \mathrm{cm}$ was used for all experiments involving water. It was prepared by passing through an ultrapure water system (Millipore, Billerica, MA).

Synthesis of the single-crystal Au seeds with a spherical or rod-like shape. The spherical seeds were prepared using a recently reported protocol. ${ }^{29}$ In a typical synthesis, the initial, CTAB-capped Au seeds were prepared by adding $0.6 \mathrm{~mL}$ of an aqueous $\mathrm{NaBH}_{4}$ solution (10 $\mathrm{mM}$ ) into $10 \mathrm{~mL}$ of an aqueous solution containing $\mathrm{HAuCl}_{4}(0.25 \mathrm{mM})$ and CTAB $(100 \mathrm{mM})$. A brown solution immediately formed upon the introduction of $\mathrm{NaBH}_{4}$. The mixture was then 
kept undisturbed at room temperature for $3 \mathrm{~h}$ to ensure complete decomposition of $\mathrm{NaBH}_{4}$ remaining in the reaction solution. Then, $2 \mathrm{~mL}$ of aqueous $\mathrm{HAuCl}_{4}$ solution $(0.5 \mathrm{mM}), 2 \mathrm{~mL}$ of aqueous CTAC solution (200 mM), and $1.5 \mathrm{~mL}$ of aqueous AA solution (100 mM) were mixed in a $20 \mathrm{~mL}$ glass vial, followed by the introduction of $100 \mu \mathrm{L}$ of the initial CTABcapped Au seeds. The reaction was continued at room temperature for $10 \mathrm{~min}$. The $10 \mathrm{~nm} \mathrm{Au}$ spherical seeds were collected by centrifugation at 14,500 rpm for $30 \mathrm{~min}$, washed with water once, and then re-dispersed in $1 \mathrm{~mL}$ of aqueous CTAC solution $(20 \mathrm{mM})$ for further use. For the synthesis of $25 \mathrm{~nm}$ Au spherical seeds, $2 \mathrm{~mL}$ of aqueous CTAC solution (100 mM), 130 $\mu \mathrm{L}$ of aqueous AA solution $(10 \mathrm{mM})$, and $50 \mu \mathrm{L}$ of the $10-\mathrm{nm}$ seeds were mixed in a $20 \mathrm{~mL}$ glass vial, followed by dropwise addition of $2 \mathrm{~mL}$ of aqueous $\mathrm{HAuCl}_{4}$ solution $(0.5 \mathrm{mM})$ using a syringe pump at an injection rate of $2 \mathrm{~mL} / \mathrm{h}$. The reaction was allowed to proceed at room temperature for $10 \mathrm{~min}$ after the injection had been finished. The final product was collected by centrifugation at $14500 \mathrm{rpm}$ for $10 \mathrm{~min}$ and washed with water once, and then re-dispersed in $1 \mathrm{~mL}$ of aqueous CTAC solution (20 mM).

The Au rod-like seeds were prepared using a seed-mediated growth method. ${ }^{30}$ Typically, 2 $\mathrm{mL}$ of aqueous $\mathrm{HAuCl}_{4}$ solution $(0.5 \mathrm{mM}), 2 \mathrm{~mL}$ of aqueous CTAB solution $(100 \mathrm{mM}), 100$ $\mu \mathrm{L} \mathrm{mL}$ of aqueous $\mathrm{AgNO}_{3}$ solution $(2 \mathrm{mM})$, and $110 \mu \mathrm{L}$ of aqueous AA solution (10 mM) were mixed in a $20 \mathrm{~mL}$ glass vial, followed by the introduction of $2.4 \mu \mathrm{L}$ of the initial CTABcapped Au seeds. The reaction was allowed to continue at room temperature for $30 \mathrm{~min}$. The product was collected by centrifugation at $14,500 \mathrm{rpm}$ for $10 \mathrm{~min}$, washed with water once, and then re-dispersed in $1 \mathrm{~mL}$ of aqueous CTAC solution $(20 \mathrm{mM})$ for further use.

Synthesis of the single-crystal Au seeds with a cubic or octahedral shape. The Au cubic seeds were synthesized using a published procedure. ${ }^{31}$ Typically, $0.2 \mathrm{~mL}$ of aqueous $\mathrm{HAuCl}_{4}$ solution (10 mM), $5 \mathrm{~mL}$ of aqueous CTAB solution (20 mM), $4.5 \mathrm{~mL}$ CTAC solution (200 $\mathrm{mM}$ ), and $200 \mu \mathrm{L}$ of AA solution (100 mM) were mixed in a $20 \mathrm{~mL}$ glass vial, followed by the introduction of $10 \mu \mathrm{L}$ of the Au spherical seeds. The reaction was allowed to continue at room temperature for $60 \mathrm{~min}$. The product was collected by centrifugation at 14,500 rpm for $30 \mathrm{~min}$, washed with water once, and then re-dispersed in $1 \mathrm{~mL}$ of aqueous CTAC solution (20 mM) for further use.

The Au octahedral seeds were prepared using a protocol similar to what was used for the Au cubic seeds, except for the amount of AA. ${ }^{31}$ It was found that the final shape taken by the Au nanocrystals was strongly affected by the concentration of AA. At low concentrations of 
AA, the formation of a thermodynamically stable, octahedral shape was favored. Typically, $0.2 \mathrm{~mL}$ of aqueous $\mathrm{HAuCl}_{4}$ solution $(10 \mathrm{mM}), 5 \mathrm{~mL}$ of aqueous CTAB solution (20 mM), 4.5 $\mathrm{mL}$ CTAC solution $(200 \mathrm{mM})$, and $60 \mu \mathrm{L}$ of AA solution $(100 \mathrm{mM})$ were mixed in a $20 \mathrm{~mL}$ glass vial, followed by the introduction of $50 \mu \mathrm{L}$ of the Au spherical seeds. The reaction was allowed to continue at room temperature for $60 \mathrm{~min}$. The product was collected by centrifugation at 14,500 rpm for $30 \mathrm{~min}$, washed with water once, and then re-dispersed in 1 $\mathrm{mL}$ of aqueous CTAC solution (20 mM) for further use.

Treatment of the Single-crystal Au seeds with aqueous $\mathrm{Na}_{2} \mathrm{~S}_{2} \mathrm{O}_{3}$ solution. Prior to the treatment, we calculated the percentage of single-crystal species in each sample of Au seeds to ensure that the number was typically higher than $90 \%$. In a standard procedure, $1 \mathrm{~mL}$ of the as-obtained seeds (spherical, rod-like, cubic, or octahedral) was mixed with $120 \mu \mathrm{L}$ of $\mathrm{Na}_{2} \mathrm{~S}_{2} \mathrm{O}_{3}$ aqueous solution (100 mM) and the mixture was aged at room temperature for $1 \mathrm{~h}$. The mixture was centrifuged at $14,500 \mathrm{rpm}$ for $30 \mathrm{~min}$ to remove excessive $\mathrm{Na}_{2} \mathrm{~S}_{2} \mathrm{O}_{3}$ and then re-dispersed in $1 \mathrm{~mL}$ of aqueous CTAC solution (20 mM) for further use.

Seed-mediated growth of Au nanocrystals. In a standard procedure, $2 \mathrm{~mL}$ of aqueous $\mathrm{HAuCl}_{4}$ solution $(0.5 \mathrm{mM}), 2 \mathrm{~mL}$ of aqueous CTAC solution $(100 \mathrm{mM})$, and $130 \mu \mathrm{L}$ of aqueous AA solution (10 mM) were mixed in a $20 \mathrm{~mL}$ glass vial, followed by rapid injection of a certain volume of the seed suspension $(100 \mu \mathrm{L}$ for the spherical seeds, $500 \mu \mathrm{L}$ for the rod-like seeds, and $250 \mu \mathrm{L}$ for the cubic or octahedral seeds). The reaction was allowed to continue at room temperature for $30 \mathrm{~min}$. The product was collected by centrifugation at 14,500 rpm for $30 \mathrm{~min}$ and then washed with water once for further characterizations.

Preparation of $\mathrm{Na}_{2} \mathrm{~S}_{\mathbf{x}}$ aqueous solution. The $\mathrm{Na}_{2} \mathrm{~S}_{\mathrm{x}}$ solution was prepared by reacting aqueous $\mathrm{Na}_{2} \mathrm{~S}$ with sulfur powders. In a typical process, $32 \mathrm{mg}$ of sulfur powders were mixed with $11.7 \mathrm{~mL}$ of aqueous $\mathrm{Na}_{2} \mathrm{~S}$ solution $(50 \mathrm{mM})$ in a $20 \mathrm{~mL}$ vial. The suspension was agitated by vortexing for about $10 \mathrm{~min}$. The vial was then capped and put in an oven held at $80{ }^{\circ} \mathrm{C}$ for $12 \mathrm{~h}$. The color of the solution turned to bright yellow once all the sulfur powders had been completely dissolved.

Instrumentation. Transmission electron microscopy (TEM) images were captured using a JEM-1400 microscope operated at $120 \mathrm{kV}$ (JEOL, Tokyo, Japan). High-resolution TEM 
images and electron diffraction patterns were obtained using a field-emission JEM-2010F microscope operated at $200 \mathrm{kV}$ (JEOL, Tokyo, Japan). The samples were prepared by dropping aqueous suspensions of the nanoparticles onto carbon-coated $\mathrm{Cu}$ grids (Electron Microscopy Sciences, Hatfield, PA) and dried under ambient conditions.

\section{RESULTS AND DISCUSSION}

The $10 \mathrm{~nm}$ Au spherical seeds with a single-crystal structure were prepared using the protocol reported in a previous publication. ${ }^{29}$ As shown in Figure 1a, these seeds exhibited a truly spherical shape and their corresponding HRTEM image and electron diffraction data confirmed their single-crystal structure (Figure 1, c and d). The seeds were treated by mixing with an aqueous solution of $\mathrm{Na}_{2} \mathrm{~S}_{2} \mathrm{O}_{3}$, followed by centrifugation and washing with water to remove the chemical species loosely bound to their surface. As shown in Figure 1b, the seeds exhibited no noticeable change in terms of both size and shape after the treatment. HRTEM imaging and electron diffraction confirmed that the seeds still showed a single-crystal structure after the treatment (Figure 1, e and f). The seed-mediated growth involved the use of $\mathrm{HAuCl}_{4}, \mathrm{CTAC}$, and AA as the Au precursor, stabilizing agent, and reductant, respectively. When the spherical seeds without $\mathrm{Na}_{2} \mathrm{~S}_{2} \mathrm{O}_{3}$ treatment were used for growth, the final products were dominated by single-crystal Au nanocrystals with a cubic shape (Figure 2a). In contrast, the use of seeds with $\mathrm{Na}_{2} \mathrm{~S}_{2} \mathrm{O}_{3}$ treatment resulted in the formation of products exhibiting a significant difference in crystallinity (Figure 2b). In particular, it is not difficult to identify twin defects in most of the nanoparticles (Figure 2c). Their corresponding electron diffraction patterns also supported the notion that most of the nanoparticles had a twinned structure (Figure 2d). ${ }^{32}$ Given that all the other experimental conditions were kept the same, it is not unreasonable to assume that the treatment of seeds with $\mathrm{Na}_{2} \mathrm{~S}_{2} \mathrm{O}_{3}$ was a critical factor responsible for the resultant difference in crystallinity for the final products.

To verify our hypothesis, we analyzed the spherical Au seeds before and after the treatment using X-ray photoelectron spectroscopy (XPS). As shown by the survey spectra in Figure 3, a and b, there were peaks for $\mathrm{Au} 4 \mathrm{f}, \mathrm{Au} 4 \mathrm{~d}, \mathrm{C} \mathrm{1s}$, and N 1s, indicating the existence of CTA units on the surfaces of both types of Au seeds. However, we noticed that the survey spectrum of the seed after the treatment also contained the S 2p peaks while no such peak was observed in the spectrum for the seeds before the treatment. Figure 3c shows highresolution XPS spectra of the S 2p peaks for the seeds after the treatment. The two peaks, 
roughly located at $168.4 \mathrm{eV}$ and $161.7 \mathrm{eV}$, could be indexed to the central (oxidized sulfur) and peripheral (reduced sulfur) atoms in the $\mathrm{S}_{2} \mathrm{O}_{3}{ }^{2-}$ anion, respectively. Compared to the results of previous XPS studies of bulk $\mathrm{Na}_{2} \mathrm{~S}_{2} \mathrm{O}_{3}$ (with binding energies of $168.4 \mathrm{eV}$ and $161.1 \mathrm{eV}$ for the central sulfur and peripheral sulfur, respectively), we noticed a blue-shift for the peripheral sulfur peak. ${ }^{33}$ Such a shift implies an electron transfer from $\mathrm{S}_{2} \mathrm{O}_{3}{ }^{2-}$ anions to the Au surface due to a strong Au-S interaction between them. In short, the XPS result implied that the thiosulfate ions had adsorbed onto the surface of the seeds during the treatment.

For the $\mathrm{S}_{2} \mathrm{O}_{3}{ }^{2-}$ anion, it contains sulfur with two different oxidation numbers: +6 and -2 . In addition, it can disproportionate into zero-valent sulfur and sulfur dioxide in an acidic medium. ${ }^{34}$ To determine which form of sulfur played the most important role, we conducted three control experiments by treating the Au seeds with other sulfur species. We chose three sulfur-containing compounds that contained sulfur with oxidation numbers of $+6,0$, and -2 : sodium sulfate $\left(\mathrm{Na}_{2} \mathrm{SO}_{4}\right)$, sodium polysulfide $\left(\mathrm{Na}_{2} \mathrm{~S}_{\mathrm{x}}\right)$, and sodium sulfide $\left(\mathrm{Na}_{2} \mathrm{~S}\right)$. The reason we chose $\mathrm{Na}_{2} \mathrm{~S}_{\mathrm{x}}$ instead of sulfur powder as the source of zero-valent sulfur can be attributed to the poor solubility of sulfur powder in water. As shown in Figure S1, the spherical Au seeds treated with $\mathrm{Na}_{2} \mathrm{SO}_{4}$ still favored the formation of $\mathrm{Au}$ nanocubes while the seeds treated either $\mathrm{Na}_{2} \mathrm{~S}_{\mathrm{x}}$ or $\mathrm{Na}_{2} \mathrm{~S}$ resulted in the formation of products with multiply twinned structures. Based on these results, we believe that the sulfur species with oxidation numbers of -2 and 0 were responsible for the change in crystallinity observed for the seed-mediated growth.

When the seed was treated with $\mathrm{S}_{2} \mathrm{O}_{3}{ }^{2-}$ anions, the chemisorbed $\mathrm{S}_{2} \mathrm{O}_{3}{ }^{2-}$ no the surface could locally interrupt the otherwise epitaxial growth involved in the deposition of $\mathrm{Au}$ on $\mathrm{Au}$. In this case, twin defects could be formed in the newly deposited layers if the added atoms could not be promptly placed in a single-crystal lattice. In addition, the sulfur atoms on the seed surface, as released by the $\mathrm{S}_{2} \mathrm{O}_{3}{ }^{2-}$ anions in an acidic medium, may change the seed surface topography, creating steps or kinks. Considering the difference in atomic radius between $\mathrm{Au}$ and $\mathrm{S}$, these steps and kinks can also induce the formation of defects if it was no longer possible to achieve cubic close packing for the Au atoms. It should be noted that these sulfur species could be desorbed from the particle surface and re-absorbed onto the newlyformed Au layer. As a result, those twin defects were formed across the entire particles rather than restricted to local regions.

We observed a similar change to crystallinity when $25 \mathrm{~nm}$ spherical seeds were used. As shown by TEM images in Figure S2, a and b, the seeds showed no noticeable change after the treatment with aqueous $\mathrm{Na}_{2} \mathrm{~S}_{2} \mathrm{O}_{3}$ solution. We then used these two types of seeds for growth 
using the standard procedure. As shown in Figure S2, c and d, single-crystal products were obtained when the seeds without $\mathrm{Na}_{2} \mathrm{~S}_{2} \mathrm{O}_{3}$ treatment were employed while the use of seeds with the treatment led to the formation of products with twin defects. Their corresponding HRTEM imaging and electron diffraction patterns also supported the notion that most of the nanoparticles had a twinned structure (Figure S2, e and f). Combined with the results from the $10 \mathrm{~nm}$ spherical seeds, it can be concluded that the size of the Au spherical seeds had no major impact on the change of crystallinity.

We then tried another type of single-crystal Au seeds--the nanorods originally reported by Murphy, El-Sayed, and other groups. ${ }^{30,35}$ As shown by TEM images in Figure 4, a and b, the nanorods showed no noticeable change in terms of both size and shape after the treatment with aqueous $\mathrm{Na}_{2} \mathrm{~S}_{2} \mathrm{O}_{3}$ solution. Figure $\mathrm{S} 3$ shows HRTEM images and electron diffraction patterns of the seeds before and after the treatment. Similar to the case of spherical seeds, no change in crystallinity for these rod-like seeds was observed after the treatment. When they were used as seeds for growth using the standard procedure, as shown by Figure 4, c and d, single-crystal products were obtained when the rod-like seeds without the treatment were employed while the use of seeds with the treatment resulted in the formation of products with twin defects. As shown in Figure S4, in particular, we notice a set of twin planes at the end of the nanorods. The presence of more than one sets of diffraction spots in the corresponding ED pattern also demonstrated the presence of twin structures.

We also tried to extend the study to single-crystal Au seeds with a cubic shape. In this case, Au cubic seeds with a single-crystal structure were prepared by following a published procedure, followed by treatment with an aqueous $\mathrm{Na}_{2} \mathrm{~S}_{2} \mathrm{O}_{3}$ solution. ${ }^{31}$ As shown by the TEM images in Figure 5, a and b, these cubic seeds showed no noticeable change in terms of both size and shape after they had been treated with the aqueous $\mathrm{Na}_{2} \mathrm{~S}_{2} \mathrm{O}_{3}$ solution. When they were employed as seeds for growth using the standard procedure, the products still exhibited a single-crystal structure (Figure 5, c and d) regardless of the treatment with $\mathrm{Na}_{2} \mathrm{~S}_{2} \mathrm{O}_{3}$ or not. Interestingly, concave side faces were developed when the cubic seeds with $\mathrm{S}_{2} \mathrm{O}_{3}{ }^{2-}$ treatment were used for growth, indicating a faster growth rate at the corner and edge sites relative to the side faces. In this case, the sulfur species were chemisorbed on the side faces, blocking the deposition of atoms onto these sites during a seeded growth process.

We observed a trend similar to the case of cubic seeds when single-crystal Au seeds with an octahedral shape were used. The octahedral seeds were prepared using a protocol similar to what was used for the Au cubic seeds, except for the amount of AA. ${ }^{31}$ Again, no noticeable 
change was observed after the $\mathrm{S}_{2} \mathrm{O}_{3}{ }^{2-}$ treatment, as indicated by the TEM images in Figure 6, a and b. Similar to the case of cubic seeds, the products had a single-crystal structure (Figure 6, c and d) regardless of the treatment with $\mathrm{S}_{2} \mathrm{O}_{3}{ }^{2-}$ or not. The Au octahedra would develop a concave structure on the side face (see Figure 6d) when the octahedral seeds were treated with $\mathrm{S}_{2} \mathrm{O}_{3}{ }^{2-}$ ions. Again, the sulfur species were chemisorbed $\mathrm{S}_{2} \mathrm{O}_{3}{ }^{2-}$ on the side faces, causing these sites to grow at a slower rate than the corner and edge sites.

According to the results from the cubic and octahedral seeds, it can be concluded that the chemisorption of the sulfur species had no selectivity toward the type of facet. They were able to bind strongly to the facets with a large area in high density and thereby completely block the growth of these facets, no matter if the facets are $\{100\}$ or $\{111\}$ type. As such, growth would only be allowed to occur at the corners and edges of a cubic or octahedral seed to generate a concave cube or octahedron. As for a spherical or rod-like seed, its surface was covered by a large number of small facets, together with a large number of corners and edges. In this case, the sulfur species might not be able to form a dense layer on the surface to block the growth from certain regions. When the Au deposited on different regions started to merge, twins and other types of defects would be introduced if the different regions were not wellaligned due to the presence of sulfur species. Taken together, the cubic and octahedral seeds would develop a concave structure on the side face when they were treated with $\mathrm{S}_{2} \mathrm{O}_{3}{ }^{2-}$ ions, whereas the spherical and rod-like seeds would develop twin defects on their surface.

\section{CONCLUSIONS}

In summary, we have demonstrated that a simple treatment of the seeds could drastically change the crystallinity or morphology of Au nanocrystals obtained through seeded growth. Specifically, single-crystal Au seeds with a spherical or rod-like shape would evolve into nanocrystals with multiple twin defects during growth if the seeds were treated with $\mathrm{S}_{2} \mathrm{O}_{3}{ }^{2-}$ ions. Treating the seeds with other sulfur-containing species, such as $\mathrm{S}^{2-}$ and $\mathrm{S}_{\mathrm{x}}{ }^{2-}$, also led to similar changes to the crystallinity. The sulfur species chemisorbed on the surface of a seed made it difficult for the newly formed Au atoms to form a single-crystal structure. As a result, twin defects were developed in the newly formed layers of Au. The twin structures were confirmed by both HRTEM imaging and electron diffraction analysis. Interestingly, the transition from a single-crystal to a twinned structure was found to be dependent on the shape of the seeds, or more precisely, the presence or absence of facets in large areas. When cubic or octahedral seeds with a single-crystal structure were used, the treatment with $\mathrm{S}_{2} \mathrm{O}_{3}{ }^{2-}$ ions 
did not cause any change to the single crystallinity. In these cases, the chemisorbed sulfur species retarded the growth of side faces, leading to the formation of cubes or octahedra with concave side faces. These methods based on surface modification for controlling the crystallinity and morphology of nanocrystals is potentially extendable to other types of noble metals.

\section{ASSOCIATED CONTENT}

\section{Supporting information}

Additional figures as described in the text (Figure S1-S4) are available. This material is available free of charge via the Internet at http://pubs.acs.org.

\section{AUTHOR INFORMATION}

\section{Corresponding Authors}

*E-mail: younan.xia@bme.gatech.edu

\section{Notes}

The authors declare no competing financial interest.

\section{ACKNOWLEDGEMENTS}

This work was supported partly by the NSF (DMR-1215034) and startup funds from Georgia Institute of Technology. As jointly supervised PhD candidates from Wuhan University, M.L. was also partially supported by the China Scholarship Council (CSC). Some work was performed at the IEN at Georgia Tech and its cleanroom facilities, a member of NNIN sponsored by the NSF.

\section{REFERENCES}

(1) Pileni, M. P. Supra- and Nanocrystallinity: Specific Properties Related to Crystal Growth Mechanisms and Nanocrystallinity. Acc. Chem. Res. 2012, 45, 1965-1972.

(2) Moriarty, J.; Vitek, V.; Bulatov, V.; Yip, S. Atomistic simulations of dislocations and defects. J. Comput. Aided Mater. Des. 2002, 9, 99-132.

(3) Marks, L. D. Experimental Studies of Small Particle Structures. Rep. Prog. Phys. 1994, 57, 603-649. 
(4) Xia, Y.; Xiong, Y.; Lim, B.; Skrabalak, S. E. Shape-Controlled Synthesis of Metal Nanocrystals: Simple Chemistry Meets Complex Physics? Angew. Chem. Int. Ed. 2009, 48, 60-103.

(5) Elechiguerra, J. L.; Reyes-Gasga, J.; Yacaman, M. J. The Role of Twinning in Shape Evolution of Anisotropic Noble Metal Nanostructures. J. Mater. Chem. 2006, 16, 39063919.

(6) Yang, P.; Portales, H.; Pileni, M.-P. Identification of Multipolar Surface Plasmon Resonances in Triangular Silver Nanoprisms with Very High Aspect Ratios Using the DDA Method. J. Phys. Chem. C. 2009, 113, 11597-11604.

(7) Grimvall, G. The Electron-Phonon Interaction in Metals; North-Holland: New York, 1981.

(8) Portalès, H.; Goubet, N.; Saviot, L.; Yang, P.; Sirotkin, S.; Duval, E.; Mermet, A.; Pileni, M.-P. Crystallinity Dependence of the Plasmon Resonant Raman Scattering by Anisotropic Gold Nanocrystals. ACS Nano. 2010, 4, 3489-3497.

(9) Chang, S. C.; Leung, L. W. H.; Weaver, M. J. Metal Crystallinity Effects in Electrocatalysis As Probed by Real-Time FTIR Spectroscopy: Electrooxidation of Formic Acid, Methanol, and Ethanol on Ordered Low-Index Platinum Surfaces. J. Phys. Chem. 1990, 94, 6013-6021.

(10) Portales, H.; Goubet, N.; Saviot, L.; Adichtchev, S.; Murray, D. B.; Mermet, A.; Duval, E.; Pileni, M.-P. Probing Atomic Ordering and Multiple Twinning in Metal Nanocrystals through Their Vibrations. Proc. Natl. Acad. Sci. USA. 2008, 105, 1478414789.

(11) Goubet, N.; Yan, C.; Polli, D.; Portalès, H.; Arfaoui, I.; Cerullo, G.; Pileni, M.-P. Modulating Physical Properties of Isolated and Self-Assembled Nanocrystals through Change in Nanocrystallinity. Nano Lett. 2012, 13, 504-508.

(12) Zheng, J.; Ding, Y.; Tian, B.; Wang, Z. L.; Zhuang, X. Luminescent and Raman Active Silver Nanoparticles with Polycrystalline Structure. J. Am. Chem. Soc. 2008, 130, 10472-10473.

(13) Polli, D.; Lisiecki, I.; Portalès, H.; Cerullo, G.; Pileni, M.-P. Low Sensitivity of Acoustic Breathing Mode Frequency in Co Nanocrystals upon Change in Nanocrystallinity. ACS Nano 2011, 5, 5785-5791.

(14) Gu, Q. F.; Krauss, G.; Steurer, W.; Gramm, F.; Cervellino, A. Unexpected High Stiffness of Ag and Au Nanoparticles. Phys. Rev. Lett. 2008, 100, 045502. 
(15) Niu, W.; Xu, G. Crystallographic Control of Noble Metal Nanocrystals. Nano Today 2011, 6, 265-285.

(16) Tang, Y.; Ouyang, M. Tailoring Properties and Functionalities of Metal Nanoparticles through Crystallinity Engineering. Nature Mater. 2007, 6, 754-759.

(17) Zhang, Q.; Xie, J.; Yu, Y.; Yang, J.; Lee, J. Y. Tuning the Crystallinity of Au Nanoparticles. Small 2010, 6, 523-527.

(18) Liu, Y.; Zhu, Z.; Liu, G.; Xu, Z.; Kuznicki, S. M.; Zhang, H. A Novel Method to Improve Crystallinity of Supported Nanoparticles Using Low Melting Point Metals. $J$. Phys. Chem. C. 2011, 115, 14591-11597.

(19) Xia, X.; Xia, Y. Symmetry Breaking during Seeded Growth of Nanocrystals. Nano Lett. 2012, 12, 6038-6042.

(20) Xiong, Y.; Chen, J.; Wiley, B.; Xia, Y.; Aloni, S.; Yin, Y. Understanding the Role of Oxidative Etching in the Polyol Synthesis of Pd Nanoparticles with Uniform Shape and Size. J. Am. Chem. Soc. 2005, 127, 7332-7333.

(21) Li, J.; Zheng, Y.; Zeng, J.; Xia, Y. Controlling the Size and Morphology of Au@Pd Core-Shell Nanocrystals by Manipulating the Kinetics of Seeded Growth. Chem. Eur. J. 2012, 18, 8150-8156.

(22) Ma, Y.; Li, W.; Cho, E. C.; Li, Z.; Yu, T.; Zeng, J.; Xie, Z.; Xia, Y. Au@Ag CoreShell Nanocubes with Finely Tuned and Well-Controlled Sizes, Shell Thicknesses, and Optical Properties. ACS Nano 2010, 4, 6725-6734.

(23) Eguchi, M.; Mitsui, D.; Wu, H.-L.; Sato, R.; Teranishi, T. Simple Reductant Concentration-Dependent Shape Control of Polyhedral Gold Nanoparticles and Their Plasmonic Properties. Langmuir 2012, 28, 9021-9026.

(24) Zeng, J.; Zheng, Y.; Rycenga, M.; Tao, J.; Li, Z.-Y.; Zhang, Q.; Zhu, Y.; Xia, Y. Controlling the Shapes of Silver Nanocrystals with Different Capping Agents. J. Am. Chem. Soc. 2010, 132, 8552-8553.

(25) Zhu, C.; Zeng, J.; Lu, P.; Liu, J.; Gu, Z.; Xia, Y. Aqueous-Phase Synthesis of SingleCrystal Pd Seeds $3 \mathrm{~nm}$ in Diameter and Their Use for the Growth of Pd Nanocrystals with Different Shapes. Chem. Eur. J. 2013, 19, 5127-5133.

(26) Fan, F.-R.; Liu, D.-Y.; Wu, Y.-F.; Duan, S.; Xie, Z.-X.; Jiang, Z.-Y.; Tian, Z.-Q. Epitaxial Growth of Heterogeneous Metal Nanocrystals: From Gold Nano-octahedra to Palladium and Silver Nanocubes. J. Am. Chem. Soc. 2008, 130, 6949-6952.

(27) Habas, S. E.; Lee, H.; Radmilovic, V.; Somorjai, G. A.; Yang, P. Shaping binary metal 
nanocrystals through epitaxial seeded growth. Nature Mater. 2007, 6, 692-697.

(28) Langille, M. R.; Zhang, J.; Personick, M. L.; Li, S.; Mirkin, C. A. Stepwise Evolution of Spherical Seeds into 20-Fold Twinned Icosahedra. Science 2012, 337, 954-957.

(29) Zheng, Y.; Ma, Y.; Zeng, J.; Zhong, X.; Jin, M.; Li, Z.-Y.; Xia, Y. Seed-Mediated Synthesis of Single-Crystal Gold Nanospheres with Controlled Diameters in the Range 5-30 nm and their Self-Assembly upon Dilution. Chem. Asian J. 2013, 8, 792-799.

(30) Nikoobakht, B.; El-Sayed, M. A. Preparation and Growth Mechanism of Gold Nanorods(NRs) Using Seed-Mediated Growth Method. Chem. Mater. 2003, 15, 19571962.

(31) Eguchi, M.; Mitsui, D.; Wu, H-L.; Sato, R.; Teranishi, T. Simple Reductant Concentration-Dependent Shape Control of Polyhedral Gold Nanoparticles and Their Plasmonic Properties, Langmuir 2012, 28, 9021-9026.

(32) Wang, Z. L. Transmission Electron Microscopy of Shape-Controlled Nanocrystals and Their Assemblies. J. Phys. Chem. B. 2000, 104, 1153-1175.

(33) Pelaz, B.; Grazu, V.; Ibarra, A.; Magen, C.; Pino, P. del; Fuente, J. M. de la. Tailoring the Synthesis and Heating Ability of Gold Nanoprisms for Bioapplications. Langmuir 2012, 28, 8965-8970.

(34) LaMer, V. K.; Dinegar, R. H. Theory, Production and Mechanism of Formation of Monodispersed Hydrosols. J. Am. Chem. Soc. 1950, 72, 4847-4854.

(35) Johnson, C. J.; Dujardin, E.; Davis, S. A.; Murphy, C. J.; Mann, S. Growth and Form of Gold Nanorods Prepared by Seed-mediated, Surfactant-directed Synthesis. J. Mater. Chem. 2002, 12, 1765-1770. 

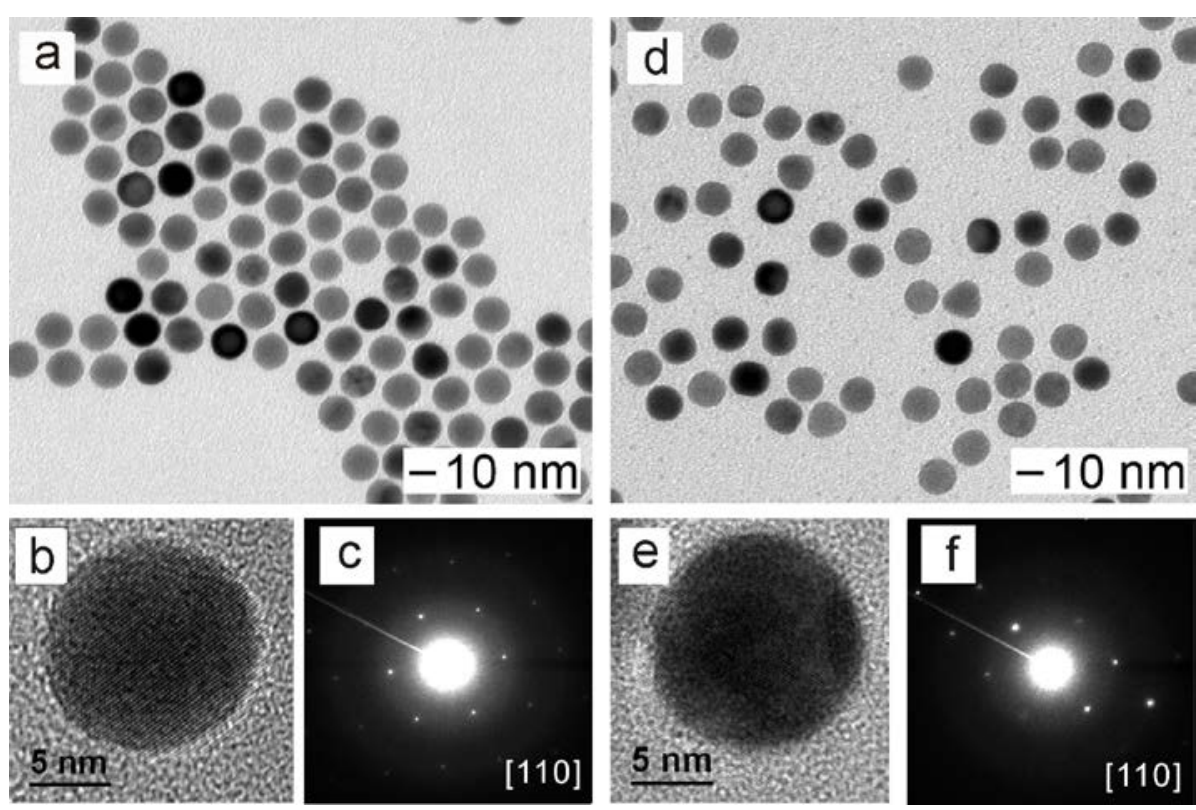

Figure 1. (a, d) TEM images of $10 \mathrm{~nm}$ Au spherical seeds (a) before and (d) after the treatment with aqueous $\mathrm{Na}_{2} \mathrm{~S}_{2} \mathrm{O}_{3}$ solution. (b, e) High-resolution TEM images and (c, f) the corresponding electron diffraction patterns of the spherical seeds (b, c) before and (e, f) after the treatment, respectively. 

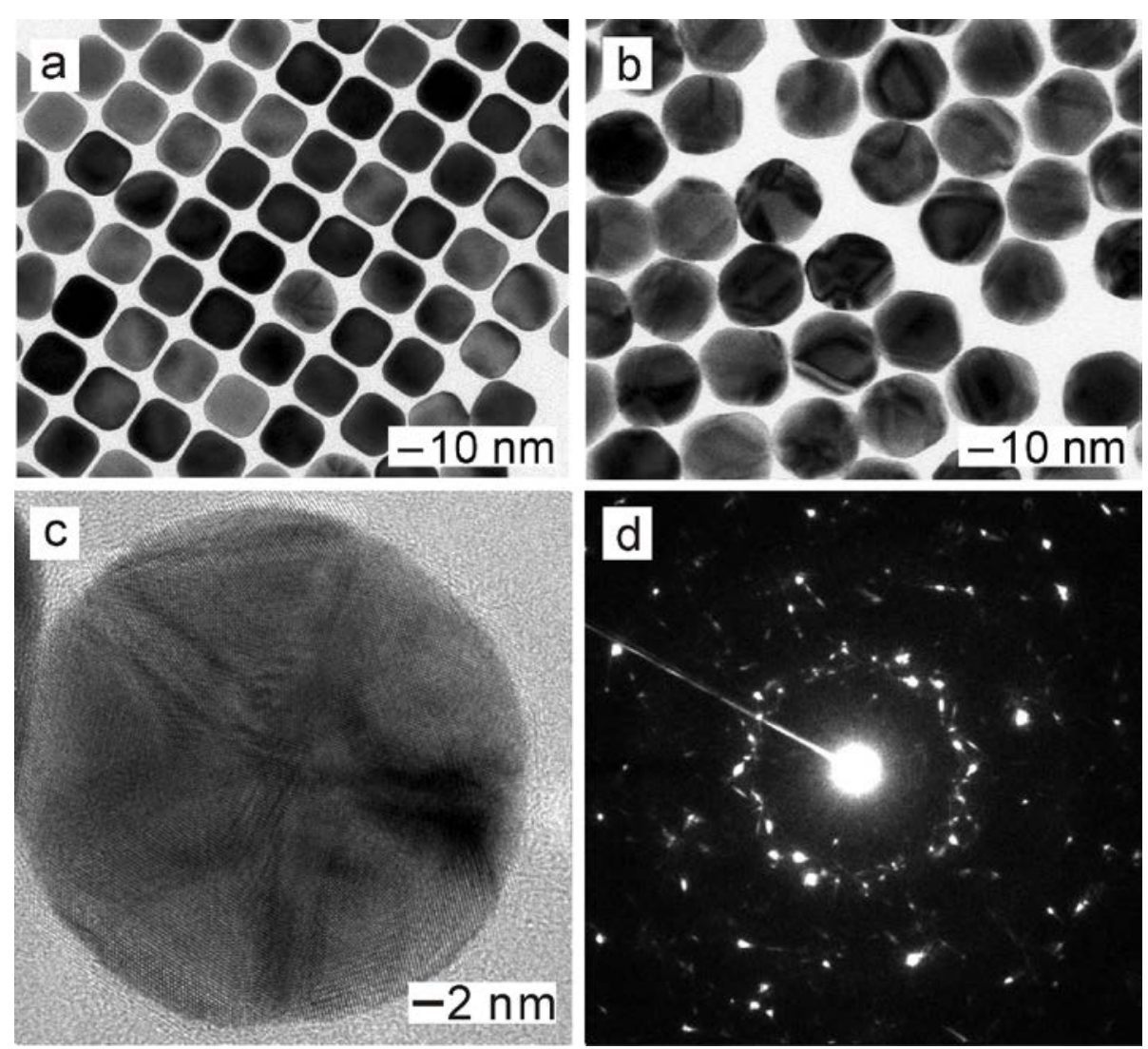

Figure 2. (a, b) TEM images of Au nanocrystals obtained using the same seed-mediated growth procedure, except that the Au seeds used for (b) had been treated with aqueous $\mathrm{Na}_{2} \mathrm{~S}_{2} \mathrm{O}_{3}$ solution. (c) High-resolution TEM image and (d) the corresponding electron diffraction pattern taken from one of the particles shown in (b). 

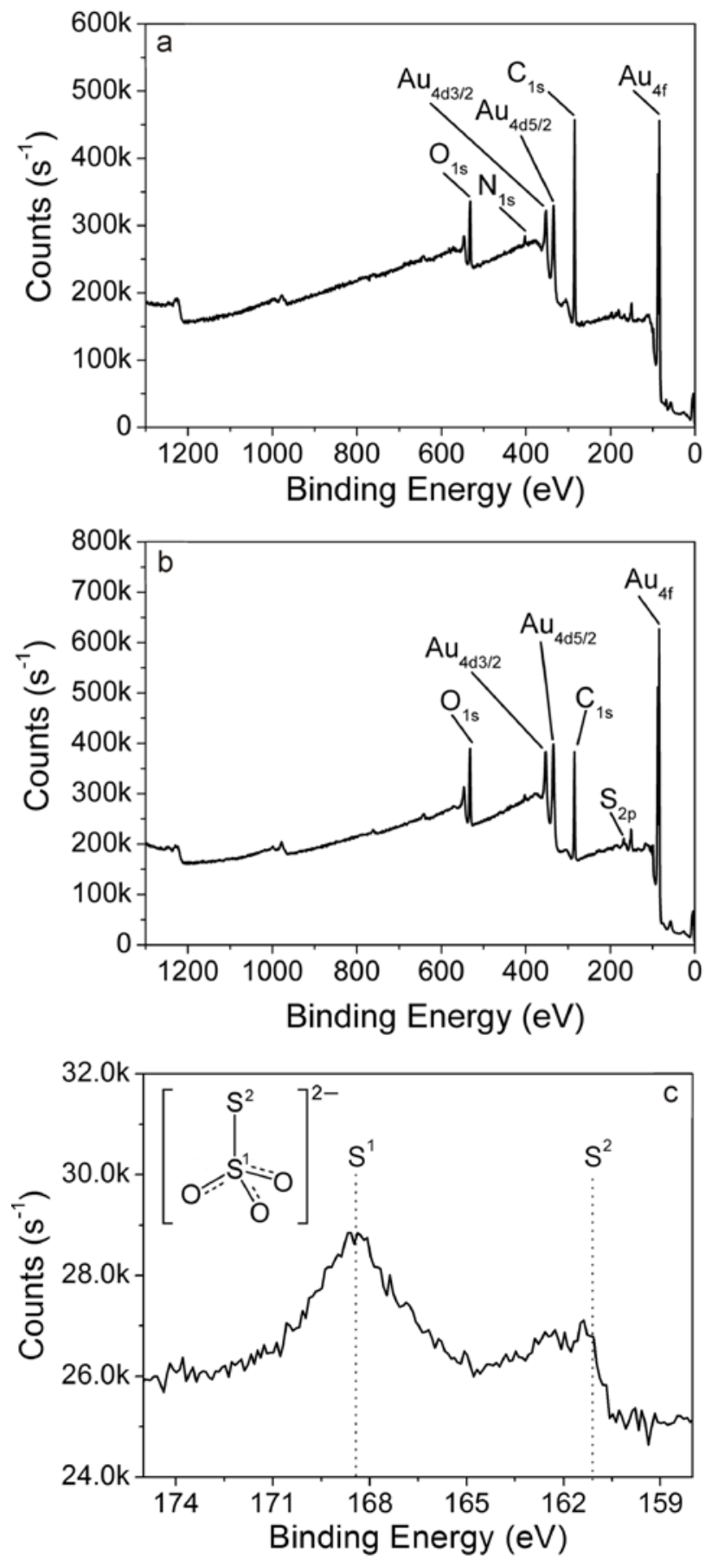

Figure 3. XPS analysis of the Au spherical seeds (a) before and (b, c) after the treatment with aqueous $\mathrm{Na}_{2} \mathrm{~S}_{2} \mathrm{O}_{3}$ solution: (a, b) survey scans; (c) $\mathrm{S} 2 \mathrm{p}$ scan of the seeds after the treatment. The dotted lines indicated the positions for the $\mathrm{S} 2 \mathrm{p}$ peaks of bulk $\mathrm{Na}_{2} \mathrm{~S}_{2} \mathrm{O}_{3}$ reported in the literature. 


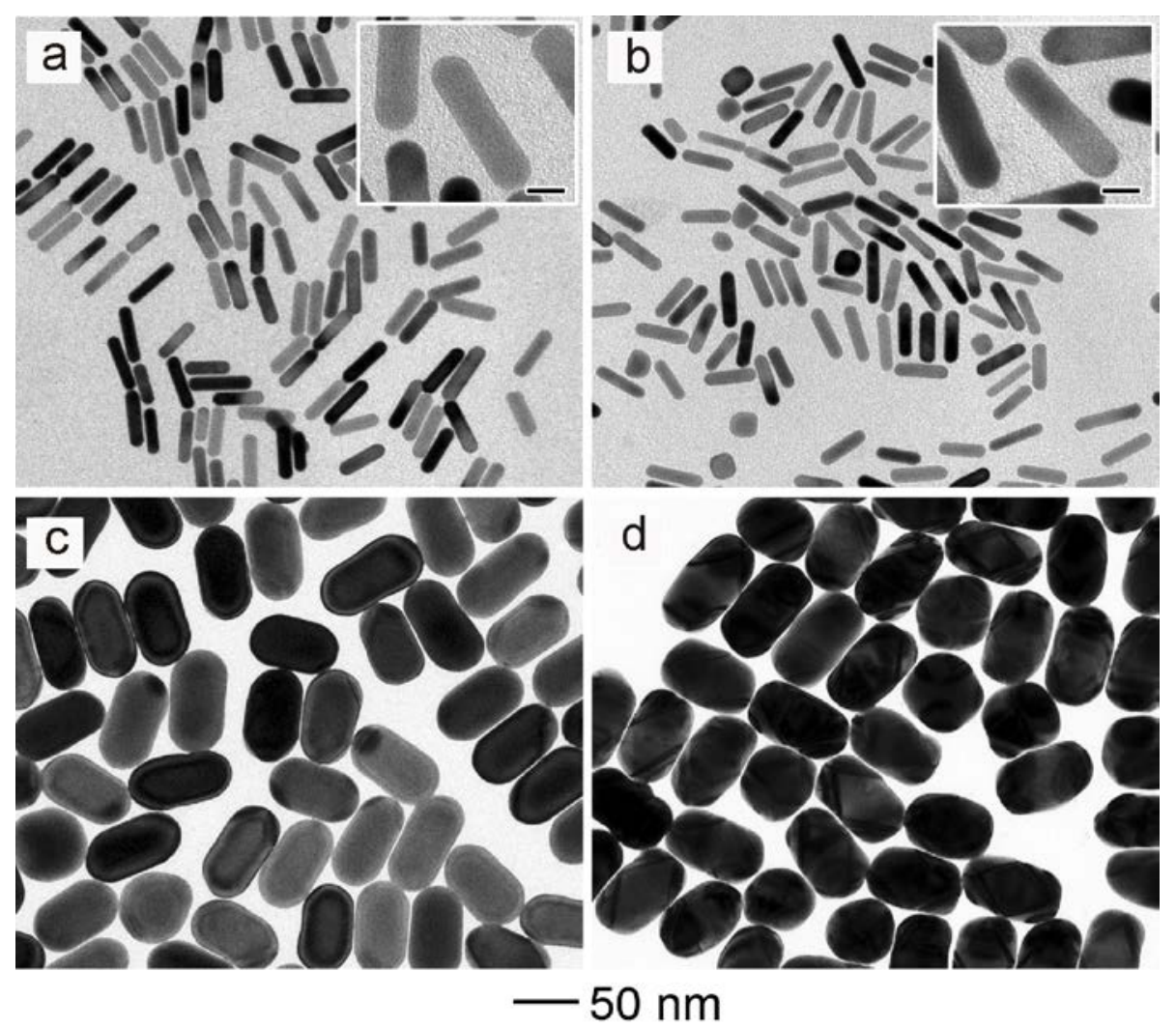

Figure 4. (a, b) TEM images of Au rod-like seeds (a) before and (b) after treatment with aqueous $\mathrm{Na}_{2} \mathrm{~S}_{2} \mathrm{O}_{3}$ solution. (c, d) TEM images of Au nanocrystals that were obtained using the same procedure for seeded growth, expect that the rod-like seeds used for (d) had been treated with aqueous $\mathrm{Na}_{2} \mathrm{~S}_{2} \mathrm{O}_{3}$ solution. The scale bars in the insets of (a) and (b) are $10 \mathrm{~nm}$. 


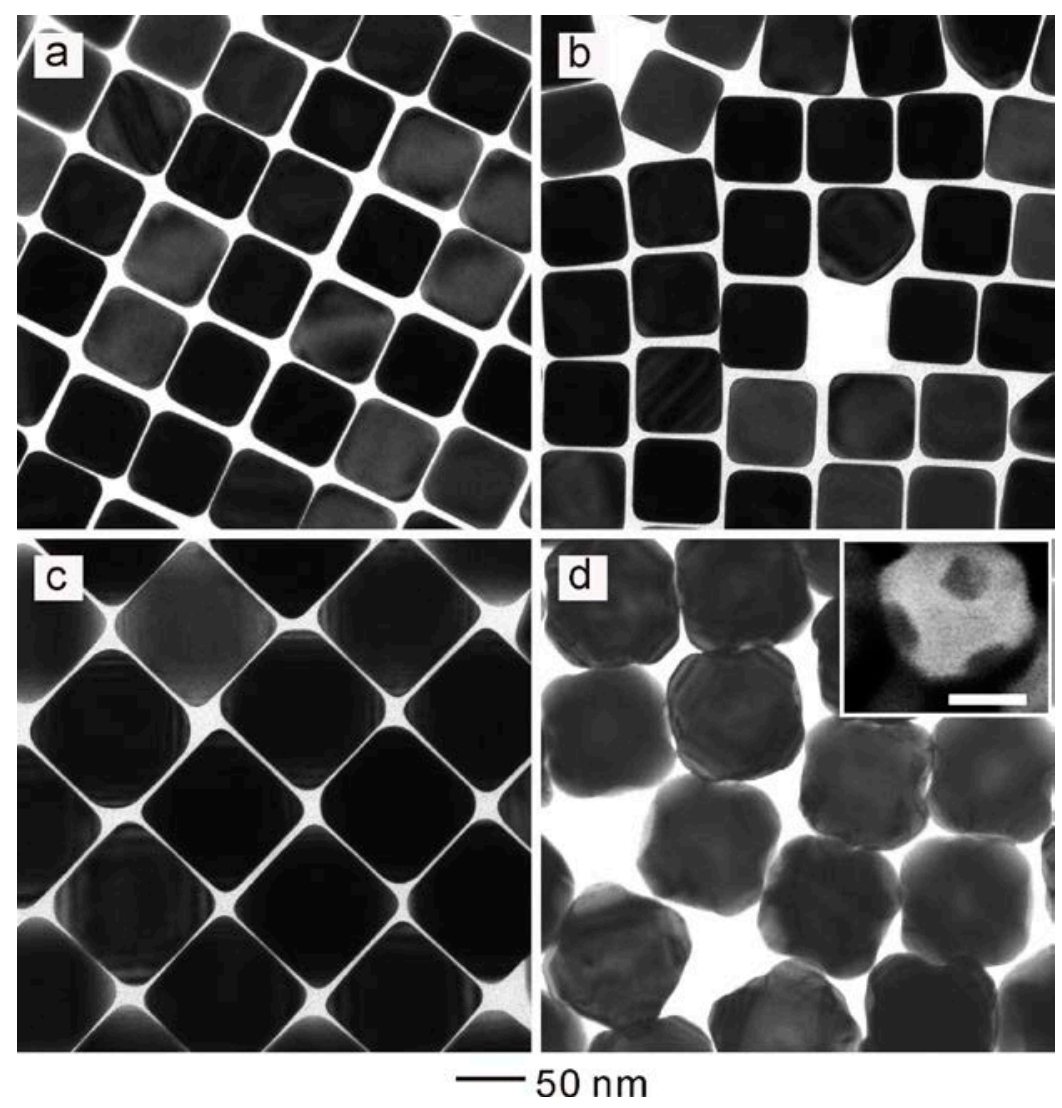

Figure 5. (a, b) TEM images of Au cubic seeds (a) before and (b) after treatment with aqueous $\mathrm{Na}_{2} \mathrm{~S}_{2} \mathrm{O}_{3}$ solution. (c, d) TEM images of Au nanocrystals that were obtained using the same procedure, except that the cubic seeds used for (d) had been treated with aqueous $\mathrm{Na}_{2} \mathrm{~S}_{2} \mathrm{O}_{3}$ solution. The inset in (d) shows an SEM image of the concave cube (scale bar: 50 $\mathrm{nm})$. 


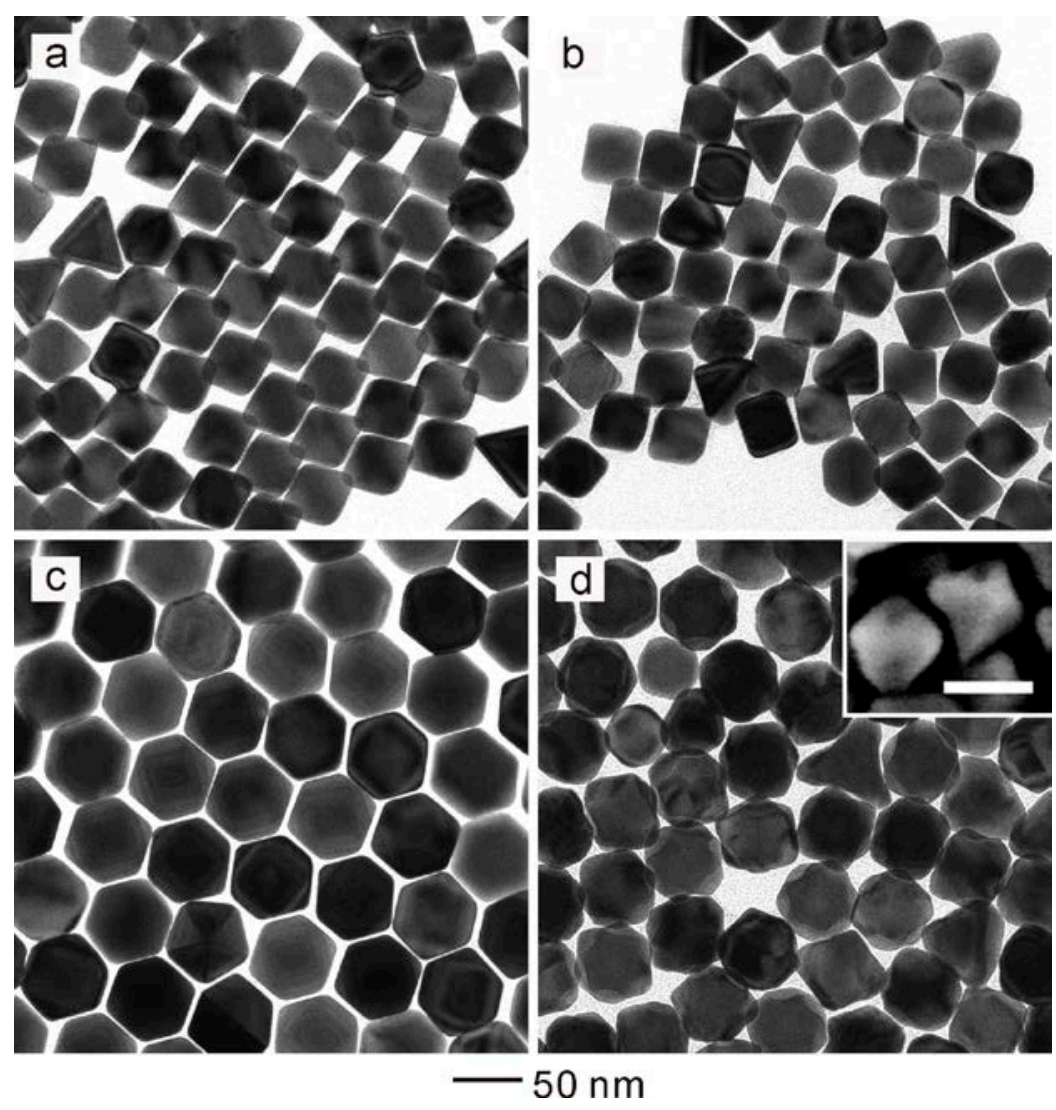

Figure 6. (a, b) TEM images of Au octahedral seeds (a) before and (b) after treatment with aqueous $\mathrm{Na}_{2} \mathrm{~S}_{2} \mathrm{O}_{3}$ solution. (c, d) TEM images of Au nanocrystals that were obtained using the same procedure, except that the octahedral seeds used for (d) had been treated with aqueous $\mathrm{Na}_{2} \mathrm{~S}_{2} \mathrm{O}_{3}$ solution. The inset in (d) shows an SEM image of the concave octahedron (scale bar: $50 \mathrm{~nm}$ ). 
TOC

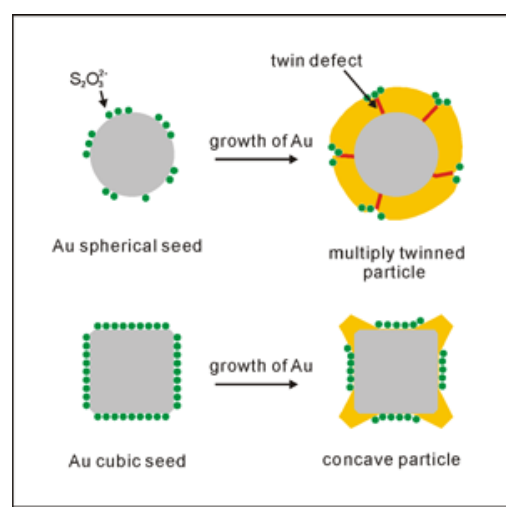

